\title{
Successful management of aberrant right hepatic duct during laparoscopic cholecystectomy: a rare case report
}

\author{
Keisuke Oyama, Shin Nakahira*, Hisataka Ogawa, Kazuya Kato, Makoto Hasegawa, Takayuki To, Ryosuke Maki, \\ Hoshi Himura, Hidemi Nishi, Nobuyoshi Ohhara, Jota Mikami, Yoichi Makari, Ken Nakata, Masaki Tsujie \\ and Junya Fujita
}

\begin{abstract}
Background: Anatomic variants of the biliary tree present challenges to surgical management during laparoscopic cholecystectomy and affect perioperative outcomes. An aberrant right hepatic duct connecting into the cystic duct is a practically important variation because of the susceptibility to serious postoperative refractory bile leakage. We report a successful case of laparoscopic cholecystectomy in the aberrant right hepatic duct of a patient diagnosed with chronic cystitis.

Case presentation: A 49-year-old man was referred to our department for treatment of chronic cholecystitis. Magnetic resonance cholangiopancreatography indicated that the cystic duct branched from the common bile duct and an aberrant bile duct connected to the cystic duct. Intraoperative cholangiography revealed that the bile duct was not confluent to the major right branch of the intrahepatic bile duct and drained a narrow area.

Preoperative magnetic resonance cholangiopancreatography had diagnostic value. Furthermore, intraoperative cholangiography with the Critical View of Safety method was paramount to achieving safe cholecystectomy based on confirmation of the biliary anatomy and the drainage area of the aberrant right hepatic duct.

Conclusion: We encountered a rare but clinically significant case of laparoscopic cholecystectomy. This case suggests that precise understanding of the anatomy and drainage area of the aberrant right hepatic duct preoperatively and intraoperatively can lead to safe cholecystectomy.
\end{abstract}

Keywords: Aberrant right hepatic duct, Laparoscopic cholecystectomy

\section{Background}

Laparoscopic cholecystectomy is currently regarded as the standard surgical treatment for cholecystitis and cholecystolithiasis. Bile duct injury continues to be a serious complication of laparoscopic cholecystectomy [1] and occasionally stems from the presence of unrecognized variants of the anatomical biliary tree $[2$, 3]. Anatomical variants of the biliary tree include aberrant right hepatic duct (ARHD), which has an incidence of approximately 5\% (1.02-35\%) [4]. An ARHD drains primarily into the common hepatic duct, common bile duct, or left hepatic duct. Notably, an ARHD rarely flows

\footnotetext{
* Correspondence: nakahira@sakai-hospital.jp

Department of Surgery, Sakai City Medical Center, 1-1-1 Ebarajicho, Nishi-ku, Sakai City, Osaka 593-8304, Japan
}

into the cystic duct, and this anatomical variant occasionally accounts for an injured ARHD during surgery [5]. A rare occurrence of ARHD draining into the cystic duct requires meticulous care during cholecystectomy, as injury can prompt postoperative bile leakage and postsurgical complications. The Critical View of Safety (CVS) technique first introduced by Strasberg is based on the precise anatomical assessment and identification of biliary tree variants $[1,5]$ and can prevent accidental biliary and vascular injuries due to uncommon anatomical variations $[1,6]$.

Magnetic resonance cholangiopancreatography (MRCP) was recently reported to be an optimal imaging modality that can provide biliary tract information and accurately distinguish the presence of biliary tree variants [7]. 
The case presented here reports the successful application of laparoscopic cholecystectomy supported by preoperative MRCP in the management of an ARHD draining into the cystic duct of a patient with chronic cholecystitis.

\section{Case presentation}

A 49-year-old male with a history of cholelithiasis presenting with right hypochondoralgia with a positive Murphy's sign was referred to our department for surgical treatment. Computed tomography (CT) without contrast media revealed a gallstone in the thickened gallbladder wall with a slight increase in the CT value due to surrounding panniculitis (Fig. 1). MRCP revealed that the cystic duct branched from the common bile duct and an aberrant bile duct connected to the cystic duct (Fig. 2, yellow arrow). The link between the aberrant bile duct and major intrahepatic biliary system was not visually apparent by MRCP. The patient was diagnosed with chronic calculous cholecystitis with aberrant bile duct flow into the cystic duct. During laparoscopic cholecystectomy, four ports were placed: a 12-mm camera port in the umbilical area by open method, $12-\mathrm{mm}$ port in the epigastric area, $5-\mathrm{mm}$ port in the right subcostal area, and a $5-\mathrm{mm}$ port at the right lateral abdomen. Due to inflammatory fibrotic adhesion in Calot's triangle, the ARHD was excessively exposed. A fundus-first technique was performed to confirm the ARHD after exposure of the inner layer of the subserosal layer at the dorsal and ventral side of Calot's triangle. ARHD drainage into the cystic duct was confirmed. Preoperative MRCP suggested it was not necessary to preserve the ARHD with the extreme narrow drainage area as it seemed to be closed at the hepatic side without communicating with the major right branch of the intrahepatic bile duct.

Intraoperative cholangiography from the cystic duct in the periphery (Fig. 3) revealed that the ARHD was not

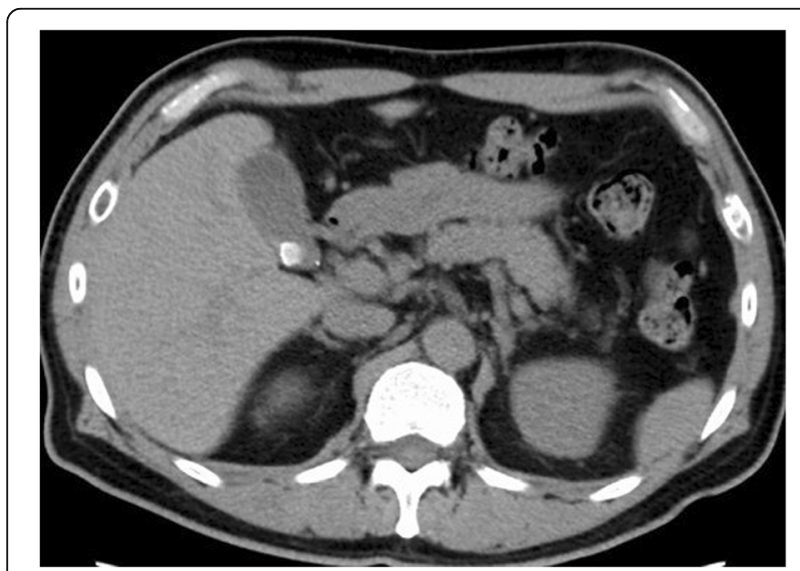

Fig. 1 Computed tomography showing calculous cholecystitis (axial sections)

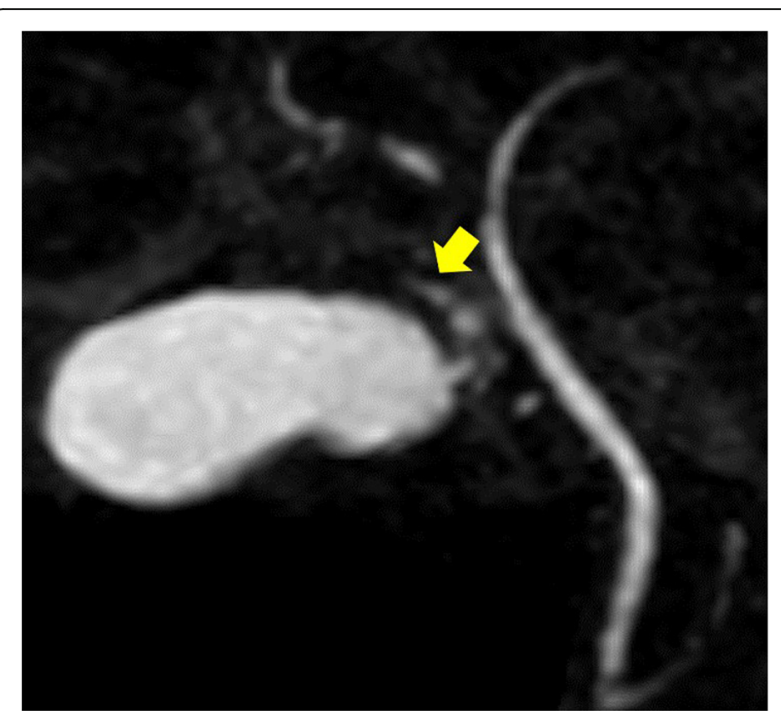

Fig. 2 Magnetic resonance cholangiography showing the cystic duct branching from the common bile duct and an aberrant right hepatic duct (arrow) connecting to the cystic duct

confluent with the major right branch of the intrahepatic bile duct and drained a narrow area, so we removed the excessively exposed ARHD. Removal and ligation of the ARHD on the hepatic side and cystic duct was performed by clipping (AESCULAP DS Titanium Ligation Clips, B Braun brand, Tokyo, Japan). An absence of bile leakage precluded any placement of drainage. The surgical movie is available online only (Additional file 1 : Video). The postoperative course was uneventful, and the patient was discharged on the third postoperative day. Follow-up MRCP showed no dilated bile duct in the liver 1 month after surgery (Fig. 4). The resected specimen was diagnosed as chronic cholecystitis. Laboratory analysis showed no abnormal increase in AST, ALT, ALP, or bilirubin 1 month after surgery (data not shown), and no abnormal symptoms 3 months after surgery. Then, his family doctor is currently following up him.

\section{Discussion}

This case supports the importance of a meticulous understanding of biliary variations during surgery. Here, the utilization of MRCP enabled us to accurately diagnose ARHD drainage into the cystic duct before surgery. In this case, employing the CVS procedure together with the fundus-first technique provided a safe and optimal view of the confluence between the cystic duct and ARHD ending in the liver bed. Furthermore, we performed intraoperative cholangiography to judge whether the ARHD should be preserved depending on the drainage area, as it is difficult to determine an accurate 


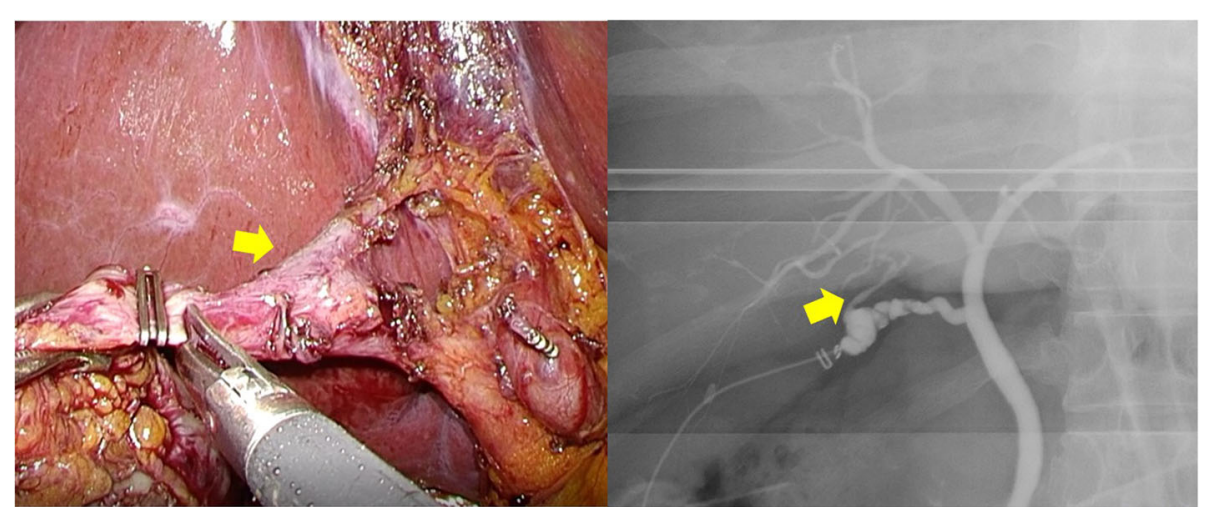

Fig. 3 Laparoscopic exploration showing a connection between the cystic duct and aberrant right hepatic duct (arrow) ending in the liver bed. Intraoperative cholangiography showing that the aberrant right hepatic duct (arrow) was neither confluent to the major hepatic duct nor responsible for draining any liver segments

corresponding drainage region. Hisatsugu et al. classified ARHD depending on the site where the ARHD and cystic duct join. According to their classification, this present case fits into the rare type $V$ (ARHD inflows into cystic duct) with a high risk of intraoperative bile duct injury [8]. Kurata et al. also reported ARHD in 40 of 506 laparoscopic cholecystectomies in reference to Hisatsugu's classification, and only one case $(0.2 \%)$ was type $\mathrm{V}$ [9]. Inappropriate removal of the ARHD is reported to be associated with recurrent obstructive cholangitis, and possibly subsequent intrahepatic lithiasis, liver atrophy, and cholangiocarcinoma $[4,10]$. On the other hand, preservation of the ARHD depends on the size. Longmire et al. described ligation of bile ducts smaller than $1 \mathrm{emm}$, but those larger than $2 \mathrm{~mm}$ or that drain one or more liver segments require reconstruction [11]. Furthermore, excessive exposure around the bile duct can induce delayed ischemic change, leading to stenosis or bile leakage [12]. In this case, intraoperative cholangiography confirmed the existence of ARHD connecting with the cystic duct and established that it was not responsible for draining any hepatic segments. From these findings, we considered that an excessively exposed ARHD should be removed to prevent delayed serious complications, and it was unnecessary to reconstruct the ARHD because of its small drainage area. Postoperative MRCP showed no dilated intrahepatic duct, which is consistent with the intraoperative judgement. Although we do not have any specific follow-up plan for a case in which the ARHD is removed, we monitored this patient and ended follow-up after no abnormal findings in the lab investigation and MRCP 3 months after surgery.

\section{Conclusion}

We present a rare but clinically significant case of ARHD during laparoscopic cholecystectomy for the treatment of chronic calculous cholecystitis. The comprehensive combination of preoperative MRCP and intraoperative cholangiography followed by the CVS method is an effective approach for the successful management of chronic cholecystitis in patients with biliary variants.

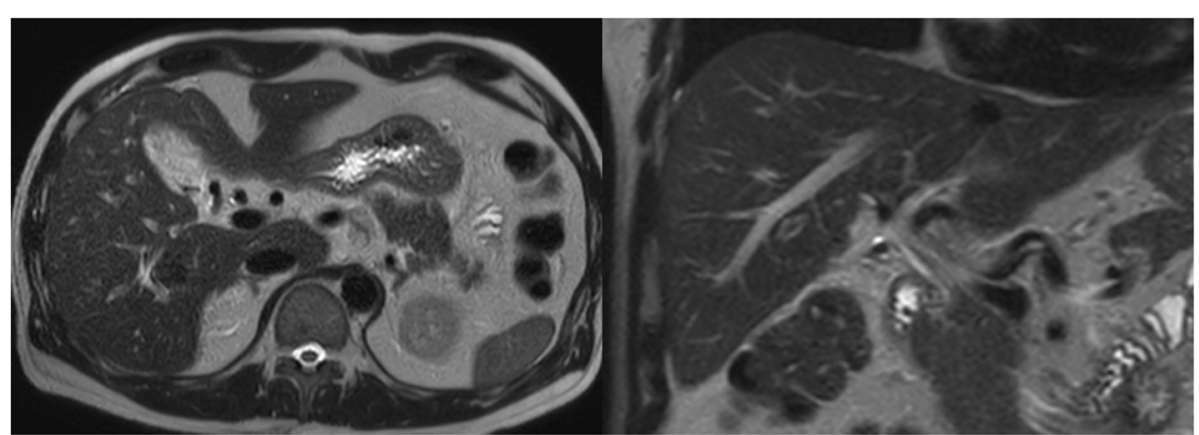

Fig. 4 Follow-up MRCP after the operation showing no dilated bile ducts in the liver 


\section{Additional file}

Additional file 1: Video Summary video of the surgery (MP4 $17983 \mathrm{~kb}$ )

\section{Abbreviations}

ARHD: Aberrant right hepatic duct; $C T$ : Computed tomography; CVS: The Critical View of Safety; MRCP: Magnetic resonance cholangiopancreatography

Acknowledgements

None.

Funding

None.

Availability of data and materials

The datasets obtained and/or analyzed during the current study are available from the corresponding author upon reasonable request.

\section{Authors' contributions}

$\mathrm{KO}$ and $\mathrm{HO}$ drafted the manuscript. $\mathrm{SN}, \mathrm{HO}$, and $\mathrm{KO}$ performed the surgery. All authors participated in the discussion and approved the final version of the manuscript.

\section{Ethics approval and consent to participate}

Not applicable.

\section{Consent for publication}

The patient provided informed consent for all data written in this study and patient anonymity was preserved.

\section{Competing interests}

The authors declare that they have no competing interests.

\section{Publisher's Note}

Springer Nature remains neutral with regard to jurisdictional claims in published maps and institutional affiliations.

Received: 26 January 2019 Accepted: 24 April 2019

Published online: 09 May 2019

\section{References}

1. Strasberg SM, Hertl M, Soper NJ. An analysis of the problem of biliary injury during laparoscopic cholecystectomy. J Am Coll Surg. 1995;180(1):101-25.

2. Doumenc B, Boutros M, Degremont R, Bouras AF. Biliary leakage from gallbladder bed after cholecystectomy: Luschka duct or hepaticocholecystic duct? Morphologie. 2016;100(328):36-40.

3. Christoforidis E, Vasiliadis K, Goulimaris I, Tsalis K, Kanellos I, Papachilea T, et al. A single center experience in minimally invasive treatment of postcholecystectomy bile leak, complicated with biloma formation. J Surg Res. 2007;141(2):171-5.

4. Sofi AA, Alaradi OH, Abouljoud M, Nawras AT. Aberrant right hepatic duct draining into the cystic duct: clinical outcomes and management. Gastroenterol Res Pract. 2011;2011:458915.

5. Lien HH, Huang CS, Shi MY, Chen DF, Wang NY, Tai FC, et al. Management of bile leakage after laparoscopic cholecystectomy based on etiological classification. Surg Today. 2004;34(4):326-30.

6. Yamashita Y, Kimura T, Matsumoto S. A safe laparoscopic cholecystectomy depends upon the establishment of a critical view of safety. Surg Today. 2010;40(6):507-13.

7. Ausch C, Hochwarter G, Taher M, Holzer B, Rosen HR, Urban M, et al. Improving the safety of laparoscopic cholecystectomy: the routine use of preoperative magnetic resonance cholangiography. Surg Endosc. 2005;19(4): 574-80.

8. Hisatsugu $T$, Yamamoto $H$, Igimi $H$, Konomi $K$, Hurusawa $T$, Jimi $M$, et al. Anomaly of bile duct including aberrant right hepatic duct with cholecystolithiasis. Rinshou Seijinbyou (J Adult Dis). 1974;4:581-6 (In Japanese)

9. Kurata M, Honda G, Okuda Y, Kobayashi S, Sakamoto K, Iwasaki S, et al. Preoperative detection and handling of aberrant right posterior sectoral hepatic duct during laparoscopic cholecystectomy. J Hepatobiliary Pancreat Sci. 2015;22(7):558-62.

10. Cai H, Kong WT, Chen CB, Shi GM, Huang C, Shen YH, et al. Cholelithiasis and the risk of intrahepatic cholangiocarcinoma: a meta-analysis of observational studies. BMC Cancer. 2015;15:831.

11. Longmire WP Jr, Tompkins RK. Lesions of the segmental and lobar hepatic ducts. Ann Surg. 1975;182(4):478-95.

12. Whitman NL, Longmire WP Jr. A clinical and experimental evaluation of the primary treatment of injuries to the common bile duct. Am J Surg. 1958; 96(2):287-95.

\section{Submit your manuscript to a SpringerOpen ${ }^{\circ}$ journal and benefit from:}

- Convenient online submission

- Rigorous peer review

- Open access: articles freely available online

- High visibility within the field

- Retaining the copyright to your article

Submit your next manuscript at $\boldsymbol{\nabla}$ springeropen.com 